\title{
Entretien avec Marc Barbut (Paris, 6 juin, 1996)
}

JMA.- Pour commencer on pourrait parler de l'époque de l'université, les études d'maths que vous avez faites.

MB.- Moi personnellement?

JMA.- Oui, j’ai calculé 1942-1946, peut être, c'est l'époque de la guerre.

MB.- Oui.

JMA.- Parce que c'est l'époque de la guerre..?

MB.- Je suis née en 1928, par conséquent en 1942 j'étais encore au lycée, j'ai passé mon Baccalauréat, qui se passait à l'époque en deux parties: une première partie une année, une deuxième partie, une deuxième année. En 1945 pour la première partie et 1946 pour la deuxième partie, voila.

JMA.- Alors, les études de maths, à quelle époque..?

MB.- Alors, j'ai commencé à faire les études de maths après ça, c'est à dire, j'ai été d'abord au lycée dans ce qu'on appelle les classes préparatoires aux grandes Ecoles en France, c'qu'on appelle en langage familier Mathématiques spéciales. Mon intention à cette époque là, c'était de devenir enseignant et préparer les concours de l'Ecole Normale Supérieure, mais j'ai été collé...

JMA.- Alors vous êtes normalien?

MB.- Non, je ne suis pas normalien, j'ai été collé, j'ai échoué aux concours.., parce que j'aimais bien les mathématiques mais dans ces concours d'entrée aux Ecoles scientifiques, la physique et la chimie jouent un rôle considérable et ça ne m'a jamais intéressé, ça me dégoûtait même profondément, surtout la chimie. De sorte que à partir de 1948 je suis passé à l'Université et j'ai suivi des études universitaires normales si on peut dire, et qui d'ailleurs convenaient plus à mon tempérament tel que je suis, les classes de préparation aux Ecoles sont des classes où on est très encadré, l'avantage c'est qu'on apprend à travailler. C'est encore des classes comme au Lycée, aucune espèce de liberté, surtout à l'époque, des ho-

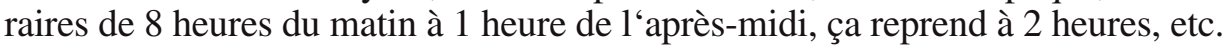
sans arrêt. C'est assez militaire comme esprit et ça ne me convenais pas. Alors 
qu'à l'Université, au contraire, c'est la grande liberté, on va aux cours au on n'y va pas, surtout à l'époque. Dans ces années là j'ai fait des études universitaires de 1948-49 jusqu' en 1951..., 1951-52-la licence était trois ans à l'époque-et le diplôme des Etudes supérieures. En ce tems-là, 1' université c'était vraiment l'université. Il y avait quelques cours auxquels on y allait ou en n'y allait pas. Et puis on travaillait seul pour le reste, seul ou en groupes avec les camarades, des petits groupes de gens qui travaillaient entre eux, qui s'interrogeaient les uns les autres. Maintenant, l'Université est devenue, surtout ce qu'on appelle le premier cycle, les premières années après le $\mathrm{BAC}$, un petit peu comme le Lycée, des petites classes, les étudiants sont encadrés, on leur fait faire des devoirs, voilà, c'est ce que j'ai vécu. Finalement le régime très libéral des études universitaires c'est arrêté en 1966, en France c'était la réforme de Fouchet, parce que ce n'était plus tenable, contenir les effectifs des gens qui ont afflue pour faire des études universitaires, quand il y a peu de monde on peut faire les choses très libéralement, mais quand il y a beaucoup de monde, c'est plus difficile.

JMA.- Et le système d'enseignement... c'était bourbakiste à l'époque?

MB.- Pas encore, à l'époque, les bourbakistes étaient professeurs à Nancy et à Strasbourg essentiellement. L'enseignement type bourbaquiste des mathématiques a commencé dans ces universités là. Mais aucun n'était encore à Paris et les professeurs qu'étaient à Paris, étaient des professeurs plus âgés, ha, ha... et n'étaient pas du tout dans cette ligne. J'ai suivi l'enseignement pre-bourbaquiste, $\mathrm{j}$ 'ai suivi un enseignement classique. Il y avait une année de mathématiques générales avec des gens comme Mr. Ceroudix qui vit encore, Mr. Dubraille qui est mort. Ensuite il y avait une année du certificat de licence, il y avait trois certificats, un d'analyse, un de physique il fallait y passer quand même et un de mécanique, qui se faisaient avec des professeurs qui sont bien oubliés aujourd'hui. Puis pour le diplôme d'études supérieures il fallait prendre une spécialisation et c'est là que j'ai pris comme spécialisation la probabilité statistique.

JMA.- Il y avait alors une spécialisation de statistique à l'université?

MB.- Voilà, elle se prenait en troisième année à l'université, et c'est là que j'ai fait la connaissance de monsieur Darmois, qui était professeur de statistique, et Paul Levy qui m'avait donné des cours sur le mouvement brownnienne. Ça était en 1950-51, c'est l'année ou j'ai connue l'Institute Statistique de l'Université de Paris, l'Institut de Poincaré, Dugê, Darmois, Fortet, Levy... essentiellement comme grands professeurs de l'époque.

JMA.- A cette époque c'est la statistique théorique..., j'ai lu dans un article de Michel Armatte que c'est 1' année où Levy-Bruhl va aux Etats Unis pour voir la façon de faire les enquêtes ... 
MB.- Bon, atteins, non, non, c'est inexact, si Armatte dit.... je ne sais pas que dit Armatte..

JMA.- C'est 1947.

MB.- Ah! Bon, en tout cas en 1950-51, premièrement Darmois commençait toujours son cours de statistique en disant «la statistique c'est pas des mathématiques», il faut que vous soyez avant tout comme des physiciens, comme des expérimentateurs, comme des gens qui mettent la main à la patte, ce qui compte c'est les données analysées, c'est le réel. Les mathématiques viennent à l'arrière encore, mais l'attitude doit être une attitude d'expérimentaliste en arrière plan, ou bien de quelqu'un qui fait des sciences d'observation, pas une attitude de mathématicien; ça il était formel-la dessus, et dans l'enseignement qui nous était donné il y avait, en effet, une partie pratique de calcul numérique, il n’y avait pas d'ordinateurs à l'époque, il y avait des petites machines...

JMA.- M. Guilbaud a dans son bureau beaucoup de trucs pour faire des expériences avec les nombres.

MB.- Voilà, on nous faisait faire des choses comme ça pour calculer une variance, du calcul numérique. La deuxième chose que je veux dire à cette égard, c'est que l'utilisation des méthodes statistiques dans l'industrie maintenant, dans les grandes entreprises comme les chemins de fer ou l'électricité etc., dans ces années là en France c'était acquis...

JMA.- Renault.., peut-être, Citroën..

MB.- Oui, le contrôle de fabrication, je crois que le livre qui a fait un peu date à l'époque..., il y en a eu deux, celui de Monsieur Desabie sur les sondages, celui qui était à 1' INSEE, sur les méthodes des sondages., qui est de ces années-là, à la fin des années 1940, début 1950, et puis il y a eu le livre de Jean Mothes, justement, sur le contrôle de fabrication qui était aussi de cette époque. Il ne faut pas dire que c'était uniquement la statistique théorique.

JMA.- Vous pouvez m'écrire les titres des livres?

MB.- De Desabie, je crois que c'était «Théorie et pratique des sondages» et l'autre c'était: «Statistique et contrôle de fabrication». Ce n'était pas l'INSEE, 1'éditeur, c'est DUNOD, je les ai dans ma bibliothèque et éventuellement je peux les emprunter, mais vous pouvez les trouver à la grande bibliothèque... Ça à commencé vraiment après la guerre, il y a eu des missions d'ingénieurs français qui sont allés aux Etats-Unis dans les années 1945-46... 
JMA.- Les ingénieurs, avant les statisticiens qui vont faire les enquêtes d'opinion..

MB.- Oui, Oui, les ingénieurs, alors, c'est un troisième domaine, les enquêtes d'opinion publique: on commencé en France en 1938, avant la guerre; à cette époque c'est Jean Stoetzel qui a créé l'Institut Française d'Opinion Publique et qui à fait, a la fin du 1938, la première enquête d'opinion par sondage, la Gallup comme on disait à l'époque..

JMA.- La méthode Gallup...

MB.- C'était, surtout, sur la crise d'été, à propos de la chute de la Tchécoslovaquie.

JMA.- En France, 1936 c'est le gouvernement du Front Populaire..

MB.- Oui.., ça a duré un an et juste après ça, parce qu'il y a eu une débâcle financière. C'était un gouvernement de centre-droite dirigé par les radicaux de l'époque: gouvernement Daladier. La crise de Munich avec les allemands...

JMA.- En 1937, il a eu une exposition universelle, j'ai vu des photos à l'Hôtel de Ville ...

MB.- Oui, je connais cette exposition..

JMA.- ...et il y avait un pavillon avec des statues portant les symboles soviétiques au Trocadero.

MB.- Oui, il y avait en bas du Trocadéro deux pavillons face à face, le pavillon soviétique avec le marteau et la faucille, un homme et une femme, et puis juste en face le pavillon allemand avec la croix gammée, l'hymne hitlérien, c'était très impressionnant..

JMA.- À 1' la exposition de l'Hôtel de Ville il y a seulement la photo soviétique....

MB.- En 1938 après les accords de Munich, il y eut la première enquête d'opinion de l'IFOP, c'était pour demander aux gens: «qu'est-ce que vous pensez des accords de Munich?» Et les gens étaient très contents, les gens étaient aveugles, parce qu'ils croyaient que la paix était sauvée, qu'il n'aurait pas de guerre, c'é- 
tait pas très joli d'avoir sacrifiés les Tchèques, mais... tout plutôt que la guerre, surtout après ce qui ce passait en Espagne.. la guerre a commencé en..

JMA.- En Espagne la guerre c'est 1936-39.

Bon,.., alors...ça c'est un peu le domaine de l'industrie et des enquêtes d'opinion publiques. Et dans le domaine de l'université, la théorie des ensembles, dans quelles années ont-elles commencé a être enseignées?

MB.- Commencent à s'enseigner.., alors.. Même dans l'enseignement que j'ai eu on commençait à en parler un petit peu..le langage, la symbolique., on 1'avait, mais ça ne devient en effet le symbole d'appartenance systématique du type bourbaquiste que en 1956, quand les premiers bourbaquistes sont arrivés à Paris.. Laurent Schwartz... une expérience personnelle. Mon premier poste d'enseignant a été au Canada, j’ai débarqué à Montréal en 1952.

JMA.- À la faculté de Math ?

MB.- Non, non, je vais vous expliquer... En 1951-52, j’ai fait le service militaire comme tous les français, c'était une année très agréable. Après, en 1952, j'ai pris un premier poste à Montréal, Canada, non pas à l'Université, c'est un peu long à expliquer; vous savez que la France entretient des Lycées français dans un certain nombre de pays étranges, alors sont des lycées. A cette époque-là, au Québec, Canada, et plus particulièrement, Québec était tenue par la hiérarchie catholique. Il n'était pas question qu'un lycée laïque de la France républicaine, vienne s'installer à Montréal. Alors on a installé à Montréal une annexe d'un collège religieux français, mais qui était lié à l'Etat, c'est l'école Stanislas qui avait une antenne, une filiale à Montréal qui joue le rôle d'un lycée français. Je l'ai choisi parce que j'était libre, j'ai enseigné aux élèves de troisième, seconde et terminale et puis j'ai fréquenté l'université, je suis allé pour voir les mathématiciens, à l'époque il n'y avait qu'une, 1'Université de langue anglaise et l'université de Montréal de langue française, et c'est-là finalement que m'on parlé de Bourbaqui.. ha, ha..

JMA.- A Montréal..

MB.- A Montréal, ils étaient très au courant certains, ils étaient en France, on allait à Nancy pour voir, alors qu'il y avait un petit département de math, mais ils allaient en pèlerinage à Nancy, Strasbourg, pour apprendre les nouvelles maths. 
JMA.- En Espagne il y a eu une reforme de éducation en 1970-71 et la première fois que j'ai vu la théorie des ensembles c'est l'année préparatoire pour aller à l'université en 1972.

MB.- Bon, écoutez. en France les choses on été à peu près les mêmes, avec quelques années, peut être, de décalage. Dans l'université ces math modernes ont été introduites au milieu des années 50, début des années 60. Simultanément, un certain nombre d'universitaires ont fortement milité pour que la même évolution se fasse dans l'enseignement du lycée, les élevés de classe secondaire, la préparation du baccalauréat et même avant, j'en ai fait partie, j'ai milité dans une association de professeurs de mathématiques des lycées, j'ai été très actif avec quelques autres, Guilbaud aussi. Le but était de moderniser l'enseignement des mathématiques pour les enfants et enseigner dès l'école primaire la théorie des ensembles et des choses comme ça. Ce mouvement a fini par avoir succès. Le Ministère a fini par nommer une commission présidé par Ms............ qui a proposé d'adopter finalement une réforme du programme comme celle que vous dites. Tout a été retardé pour les événements du mai 1968, année de grande pagaille.. cette reforme c'est introduite début 1969-70 dans l'enseignement des lycées en France,... mais ça a été finalement une catastrophe.

\section{JMA.- En Espagne aussi.}

MB.- Et alors, avec le recul, je comprends parfaitement, c'était fou de faire ça à l'époque. Pourquoi? Parce que c'était une époque où arrivaient dans les lycées des classes nombreuses d' élèves, parce qu'il y a eu le boom des naissance après la guerre, par contre, les générations d'où proviennent les profs, étaient des classes maigres, c'était les gens qui étaient nés comme moi, entre deux guerres à une époque où la France était très malthusienne et, d'autre part, il y avait très peu de vocation pour être enseignant, surtout en mathématiques. Les gens qui faisaient des études de mathématiques devenaient ingénieurs, c'était l'époque de la grande expansion économique, et il y avait un tel appel d'ingénieurs partout, que les professeurs d'enseignement étaient où bien des laisser pour contre, où bien des gens qui avaient vraiment la vocation. Les gens intelligents, travailleurs qui réussissaient en sciences d'abord sont devenus ingénieurs, s'ils ne pouvaient pas devenir ingénieurs, ils essayaient de devenir médecin, et s'ils ne pouvaient pas devenir médecins, alors ils se tournaient vers l'enseignement, alors, ç' était ça la hiérarchie. Alors, ces gens-là qui avaient été formés à l'ancienne méthode, qui avaient des classes pléthoriques, et qui n'étaient pas assez nombreuses pour enseigner, ont fait des efforts pour apprendre des choses qu'ils ne connaissaient pas, mais on enseigne bien les choses que lorsqu'on connait les choses intimement, c'était du plaqué, c'est devenu une caricature de l'enseignement que quelques années après, assez rapidement, des la fin des années soixante-dix... 
JMA.- En Espagne c'est un peu la même chose parce que je me rappelle que je n'appris presque rien, peut être parce que les profs ne connaissaient pas bien le sujet, parce qu'ils ont été formés dans les mathématiques classiques..

MB.- C'est ça, c'est ça le problème, ils connaissaient mal, ils enseignaient mal...

JMA.- Bon, à quel moment commence la relation avec les sciences sociales?

MB.- Alors, pour moi, donc. J'ai fait mes études jusqu'en 1951 à la Sorbonne, des mathématiques, après ça le service militaire, après deux ans à Montréal et je suis rentré en France en 1954 pour enseigner dans un lycée. Je suis allé voir Paul Lévi qui m'a dit: «A mon avis, ne fais pas comme moi, commence par passer l'agrégation de mathématiques parce que sinon vous ne ferez jamais carrière à l'université. Je ne suis pas prof à l'université.. »

JMA.- Ah! il n'était pas prof a l'université?

MB.- Non, il était prof à l'École Polytechnique, et donnait des cours à l'université mais comme çà, comme vacataire, parce qu'il n'y avait pas tous les sacrements, hé, hé.., bon. Levi m'a donné le conseil et c'est que j'ai fait. J'ai préparé l'agrégation en 1954-55 et je suis passé. Puis Darmois m'a dit: «il y a des choses à faire ailleurs, du côté des sciences humaines et j'essayerai de vous faire rentrer au CNRS le plus vite possible en tant chercheur». Georges Darmois, qui a été le grand-père de la statistique, est mort tôt, en 1960; évidemment j'ai vu Jean Mothes à l'époque. Si je me suis orienté vers ces études-là, c'est que mon père avait été à l'université en 1928, il était extrêmement cultivé, il était ingénieur et il avait beaucoup d'informations; il s'intéressait à ce type de choses. I avait une très grande bibliothèque et lui aussi m'avait dit que du côté de la statistique, qu'il connaissait bien, il avait lu des textes dans l'industrie papetière; et puis il était très averti sur la recherche opérationnelle après la guerre: les méthodes de mathématiques pouvaient s'appliquer aux problèmes de gestion économiques; donc il m'a orienté un peu vers çà, Jean Mothes, et Lévi aussi, voilà çà c'est fait un peu comme çà, et puis j'avais lu aux Etats Unis et au Canada un bouquin du ............. sur la théorie des jeux. Çà m'a intéressé, j'ai vu qu'il y avait beaucoup de choses à faire de ce côté-là. J'ai encore été prof de Lycée pendant un an en 1955-56. En 1956 j'ai été pris comme chercheur au CNRS. Darmois m'a dit: «Puisque vous vous intéressez un petit peu aux sciences humaines, à la théorie des jeux, à la recherche opérationnelle, il y a un homme qui doit conduire vos travaux et vous faire travailler: c'est George Guilbaud.» Alors, il m'a placé sous la dépendance scientifique de Guilbaud, que j'ai connu en 1956, et j'ai commencé, çà fait 40 ans. 
JMA.- Guilbaud était déjà ici, à...?

MB.- ...à l'Ecole des Hautes Etudes. Il y était depuis un an ou deux, il est rentré en 1955 ou 1954 ... Il y avait beaucoup de choses à faire. Il était ravi de me voir arriver parce qu'il était tout seul. Bon, il y avait lui et Madame Pétrosévitch... j'ai été comme ça deux ans au CNRS travaillant, dans le cadre de ce qui n'était pas encore le centre de mathématiques sociales, avec Guilbaud. Au CNRS, je n'ai pas voulu y rester parce que chercheur à temps plein, pour moi çà n'a aucun sens, c'est de la folie; sauf pour des individus exceptionnels, mais il faut avoir d'autres activités. Donc je suis retourné à l'enseignement; j'ai dit: «je reviens à l'enseignement.» J'ai fait de nouveau deux années au Lycée, tout en continuant à être dans le centre des mathématiques sociales. Puis, début 1960 j'ai été nommé assistant, ce qu'on l'appelait chef de travaux ici à l'Ecole des Hautes Etudes en Sciences Sociales. En 1962, j'ai été élu directeur d'études ici à l'Ecole. Alors je vais raconter toute ma vie, hé, hé.. J'ai toujours fait plusieurs choses à la fois. Ma fonction principale, disons à partir de 1960, ça a été à l'Ecole des Hautes Etudes, j'ai été élu directeur en 19 62, j' ai été élu très jeune à 34 ans. Je ne suis pas sûr de ne pas avoir le record, même encore aujourd'hui. J'ai eu de la chance parce que j'ai présenté des choses dont on avait besoin à cette époque, les générations actuelles ont beaucoup moins de chance. Ma fonction principale était ici, mais en même temps, j'ai donné des cours ailleurs, des cours puis occupé des fonctions. Dans ces années-là et jusqu'en 1966-67, j'ai donné des cours à l'Institut Statistique de Paris, qui était des cours pour les étudiants en statistique, sur certains sujets d'algèbre et de combinatoire. Egalement de 1962 à 66, j'ai été professeur à l'INSEE..

\section{JMA.- Ah! à l'INSEE.}

MB.- qui commençait tout juste au moment où Edmond Malinvaud a pris la direction, le statut de l'école a été créé, et Malinvaud m'a demandé d'être prof de math générales, un petit peu pour les étudiants qui ne venaient pas des grandes écoles; voilà, en 1966, il y a eu une réforme des études d'enseignement supérieur dans les facultés, des systèmes libéraux, des licences qu'on préparait en quelques certificats. Il y a eu une restauration en 1966 qu'on a appelé la réforme Foucher, dont les conséquences directes ont été les événements de 1968, parce que avec un régime transitoire, les français aiment bien les changements, ils sont toujours pour le changement, mais quand le changement arrive tout le monde est contre...., même s'ils ont voté pour, ha, ha..., c'est comme çà. Donc en 1966, il y a eu une réforme assez profonde des programmes de mathématiques et de statistique, qui n'existait pas pratiquement avant, pour les étudiants en sociologie, en psychologie et en philosophie; c'a été justement sur l'influence de Jean Stoetzel. Il faut que les étudiants en sociologie aient une formation non seulement statistique, les recettes statistiques, mais qu'ils aient des arrières-points ma- 
thématiques.., et le calcul des probabilités. Voilà, çà a été introduit, c'était obligatoire dans les enseignements de premier cycle de psychologie, en sociologie et philosophie. Il fallait des gens pour enseigner et c'est là qu'on m'a demandé d'enseigner à la Sorbonne, d'assurer des cours au Collège de la Sorbonne... en 1966... Tout en restant à l'Ecole, j'ai développé un certain nombre d'activités; çà a commencé dans des conditions très héroïques, parce que j'étais tout seul avec un assistant et il y avait à scolariser 1600 étudiants.

JMA.- La folie!

MB.- C'était la folie. Alors, il avait des petits groupes, déjà, et il fallait coordonner tous ces gens-là. Je faisais encore une cours magistrale dans l'amphithéâtre, mais il n’y avait aucun amphithéâtre assez grandi ... hé, hé!

JMA.- 1600 personnes !

MB.- 1600. Je faisais cours en amphithéâtre pour 400 étudiants, et les autres me voyaient dans d'autres amphithéâtres sur des écrans de télévision.... Mais çà a bien marché, finalement. Il y avait beaucoup d'efforts, c'est moi qui avait pris aux étudiants; on pouvait craindre un rejet des mathématiques par les étudiants en «socio» et en «psycho», mais pas du tout. Par contre, pour les révolutionnaires de 68 , malheureux, les mathématiques, c'était la réaction..

JMA.- Ils n'aimaient pas les maths?

MB.- Ah! non les mathématiques, c'était la sélection.

JMA.- En Espagne, c'était le contraire, les étudiants les plus révolutionnaires étaient les étudiants de sciences, de maths, de physique, des gens très cultivés qui avaient beaucoup d'intérêt pour la philosophie.

MB.- Oui, oui, ça c'est vrai, mais je parle de choses différentes, de l'attitude visà-vis de l'enseignement des mathématiques des étudiants en sciences humaines. Dans leur majorité, ils acceptaient l'enseignement des mathématiques, mais la minorité activiste qui a tout foutu en l'air, je l'ai déploré tout à fait, la minorité activiste considérait, et finalement a gagné de ce point de vue-là: que cet enseignement mathématique était l'enseignement par lequel on faisait ce qu'on appelle en français la sélection, ce qui n'était pas entièrement faux.

JMA.- De toutes façons, en Espagne il y a un problème très fort à ce sujet, je ne sais pas pourquoi, il y a une division très forte entre sciences et ce qu'on appelle lettres, il y avait le baccalauréat de sciences et le baccalauréat de lettres.. 
MB.- Oui, c'est pareil ici.

JMA.- C'est absolument stupide, parce que pourquoi ne pas étudier les maths quand on fait de la philosophie et pourquoi ne pas étudier la littérature, quand on étudie les mathématiques? Les étudiants de sociologie voient les racines carrées et commencent à avoir peur, c'est stupide, je ne sais pas pourquoi les choses ont été faites ainsi, c'est un mystère pour moi..

MB.- Oui, c'est comme çà depuis longtemps, et pas seulement en Espagne ou en France, voilà, depuis cette époque j'ai été à la fois aux Hautes Etudes et à l'Université..Paris V premièrement, Paris IV ensuite...

JMA.- À l'époque, ici, à l'Ecole, il y avait Guilbaut.. et Lévy-Strauss était ici?

MB.- Si, il était ici, à la fois au Collège de France et à l'Ecole.

JMA.- Et Lacan?

MB.- Non.

JMA.- Et Greimas?

MB.- Greimas oui, hé, hé.., Dans les années qui ont précédé 1966, disons, les deux décennies de 1960-70, c'est l'enseignement de Guilbaut et moi.

JMA.- C'est l'époque de l'article de la revue «Temps modernes» sur le structuralisme, 1967, peut-être.

MB.- Avant, j'ai fait un article sur la théorie des jeux..

JMA.- Celui de Machiavel?

MB.- Machiavel, c'est passé dans les Annales.., bon, enfin.. on avait commencé.., on agissait de trois façons dans la mathématisation des sciences humaines. La première façon c'était en pratiquant, en allant dans les séminaires, justement, en intervenant à la demande de Lévi-Strauss, de certains historiens, Raymond Aron, pour faire la théorie des jeux, bon. Deuxièmement —il y avait quatre choses - on faisait ce qu'on avait organisé en séminaire, çà a duré une dizaine d'années, çà a marché très bien, un séminaire qui se tenait ici à l'Ecole des Hautes Etudes, pas dans ce bâtiment parce qu'il n'existait pas encore, à la Sorbonne en fait..., un séminaire sur la modélisation mathématique en sciences so- 
ciales; soit des gens qui était comme nous dans ce bâtiment, qui travaillaient avec les gens des sciences sociales venait exposer ce qu'ils faisait, leurs travaux., c'était vraiment..., il y avait pas mal de monde qui le fréquentait. J'étais forcé de publier les actes de ces séminaires, des séances deux fois par mois, qui étaient diverses: sur les relations de parenté, la linguistique, les jeux. et çà a créé, au moins à Paris, un milieu de gens de sciences sociales qui avait l'habitude de fréquenter ces mathématiciens.

Le troisième type d'activité, c'était une activité alors plus pédagogique, à un moment où on a vu arriver cette fameuse réforme de 1966. Il fallait bien des enseignants pour enseigner à des psychologues et à des sociologues les mathématiques.... ce n'est pas comme enseigner aux étudiants de maths; bon, nous avons organisé des stages de formation pour des enseignants et où venait principalement d'ailleurs des sociologues ou des psychologues, des géographes, des philosophes, qui venaient comme çà.... sur l'algèbre binaire, le calcul de probabilités., et les gens venait de province, soit de Paris, soit des universités de province, et on avait comme çà tout un réseau de correspondance: Aix-en-Provence, Toulouse, Grenoble, Lille, les grandes universités, Bordeaux... Et puis la dernière forme d'action était la publication d'une revue qui a commencé en 1962, «Mathématiques et sciences humaines», qui était au début beaucoup plus tournée vers les problèmes pédagogiques qu'actuellement. Au début, c'était bien d'avoir des articles de gens qui enseignent les maths à des psychologues et des sociologues, de fournir la matière; on a publié une collection de livres... Voilà, alors en gros... D'ailleurs ce mouvement avait préparé la réforme de 1966 des universités; alors, le centre ici, au début, c'était Guilbaud lui-même, moi, madame Pétrosevich et puis, il y avait des gens qui ont disparu maintenant, qui sont partis ailleurs, ensuite. Les gens qui sont vraiment restés, il y a Rosenstiehl qui date de cette époque-là, vers 1965 il est rentré.

\section{JMA.- C'est l'actuel directeur du CAMS?}

MB.- Voilà, c'est le directeur du Centre, il est rentré à ce moment-là, Bruno Leclerc quelques années après, vers 1968, Jean Petitot..

JMA.- Mais aujourd'hui, le centre est plus orienté vers 1'informatique?

MB.- Oui, oui, c'est vrai.

JMA.- Parce qu'il me semble que c'est un mouvement des années soixante-dix.

MB.- Oui, c'est vrai, Qu'est ce qui s'est passé ensuite? Dans les années 1970, ce que j'ai vécu, l'affaire qui m’intéresse, l'utilisation des méthodes mathéma- 
tiques dans les sciences sociales, il s'est passé un triple phénomène qui a été assez catastrophique. Le premier est un phénomène institutionnel, postérieur, conséquence directe des événements de 1968 en France; il y a eu une réforme universitaire, ce qu'on a appelé la loi Edgar Faure, qui a été votée en 1968 à l'unanimité. Les communistes.., les lepénistes à l'époque n'existaient pas.. Quand on vote une loi à l'unanimité c'est qu'elle est très mauvaise bon., hé, hé, le consensus.. En tout cas, cette loi a été votée à l'unanimité, elle a consacré 1'émiettement des petits groupes, sociologues, mathématiciens, latinistes... Et alors ces petits morceaux, c'étaient des morceaux qui eux-mêmes étaient des fractions d'une discipline. Chez les psychologues de Paris, il y a eu trois morceaux, parce que les gens ne s'entendaient pas, il y avait des différences de caractère politique; chez les historiens 4 morceaux, chez les philosophes., bon, pratiquement dans toutes les disciplines, les gens se sont divisés en plusieurs morceaux, il y avait des gens de gauche, de droite, et puis d'ultragauche .... on a reconnu les morceaux par université, par affinité; c'est comme ça que ce sont faites les universités, elles se sont créées soit sur un critère purement politique —c'est le cas de Paris VII et Paris IV_- extrême-gauche à Vincennes, extrême-droite à Paris IV...

\section{JMA.- Meusnier est à Vincennes?}

MB.- Voilà, bon, ces universités se sont constituées comme çà, sur des bases qui n'ont vraiment rien de scientifique; il y avait des choses à faire, disciplinaires. Le gros des psychologues et des sociologues, on les a pris à l'université qui s'appelle encore Paris V, IV avec la Médecine. Pourquoi? La raison était politique, car c'est le Ministère qui l'a voulu, c'étaient les étudiants les plus agités, et par contre la faculté de médecine était bien calme; c'était les mettre dans un cordon sanitaire. Donc ces universités étaient multidisciplinaires, ces féodalités n'ont plus travaillé ensemble. La «psycho», c'était extraordinaire, j'ai connu ces facultés de lettres à la Sorbonne d'avant 68, il y avait des assemblées, tous les professeurs étaient là, toutes les disciplines se voyaient et on parlait, et puis entre disciplines voisines on travaillait ensemble, dans le même groupement, les psychologues, les sociologues, les sciences de l'éducation, les mathématiciens; donc j'étais le petit mathématicien, parce que j'ai travaillé avec eux, on discutait les programmes, les choses des enseignants, tout ça se faisait en collaboration interdisciplinaire. A partir du moment où chacun était dans son petit coin, fini; les psychologues et les sociologues ne se sont plus parlé pendant 15 ans.....

Alors les mathématiciens, on a fait nous aussi notre petit groupe à part —on n'avait pas d'autre solution — avec moins de liaison interdisciplinaire qu'avant. La loi de 1968, très vive face aux connexions interdisciplinaires, a fait exactement le contraire, a exorbité les aspects idéologiques. Avant de commencer un cours, ce sont les étudiants qui vont décider ce qu'il faut étudier, je caricature, mais...

EMPIRIA. Revista de Metodología de Ciencias Sociales. N. ${ }^{\circ}$ 24, junio-diciembre, 2012, pp. 191-208. ISSN: 1139-5737 
JMA.- En Espagne çà a été à peu près la même chose; les étudiants ont décidé de ne pas étudier les statistiques, ils préféraient étudier le marxisme...

MB.- Ce mouvement a donc beaucoup freiné les rapports que nous avions entre mathématiques et sciences sociales. Deuxième chose qui s'est passée, qui n'est pas institutionnelle, çà a été.. -la deuxième et la troisième chose sont simul-

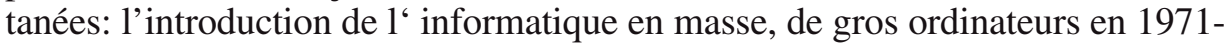
72, ici dans le bâtiment. L'informatique arrive en sciences humaines et la méthode de Benzecri, les méthodes d'analyse des données, les analyses factorielles avec des programmes d'ordinateur. A partir de ce moment le sociologue moyen, l'historien moyen, le géographe moyen, même l'économiste moyen ont dit: «Pourquoi se casser la nénette à étudier tel ou tel problème en collaboration avec un statisticien si on comprend..

JMA.- Braudel aussi?

MB.- Pas Braudel, mais François Furet, Ladurie.., je les ai vu avec les cartes perforées sous le bras descendre au sous-sol, et allez, hop! faire tourner le programme et le numéro de programme, et puis la vérité est sortie, et non, plus de réflexion. Dans les années 60 au contraire, il y a eu des rapports avec LévyStrauss, avec.. des historiens comme Da Silva, même Ladurie, où on réfléchissait et où on essayait de formuler des hypothèses, de fabriquer un modèle. Les articles dont vous parliez tout à l'heure: «Machiavel et les maths», à partir de 1970 c'est impensable!

JMA.- J'ai sur ma table «Le soldat américain», fait pendant la guerre et publié en 1948, peut être, juste après la guerre; çà a été fait pendant la guerre, et çà commence en disant: «Bon, mesurer les attitudes est toujours problématique, parce que qu'est-ce qu'une attitude? Il y a un esprit critique. Il utilise le modèle statistique, mais il présente le titre en disant: «Bon, il y a un problème théorique derrière, nous allons travailler avec ça parce qu'il n'y a pas d'autre possibilité pour le moment..

MB.- Oui

JMA.- Çà va disparaître dans toutes les études, pendant les années 1970, chez nous.

MB.- Complètement. 
JMA.- Et ce sont les américains qui vont parler à tout le monde de la méthode statistique, mais il y a encore un esprit critique, «nous allons utiliser cette méthode parce qu'il n y en a pas d'autre... »

MB.- En France çà a été une catastrophe, çà a coïncidé a peu près au moment où ce bâtiment a été construit, après la guerre. La Maison des Sciences de l'Homme a été faite pour collaborer entre les disciplines, et çà a été le contraire; pour nous qui étions un petit groupe qui faisait de la mathématique sociale, L'Ecole des Hautes Etudes était dispersée entre plusieurs rues de Paris, et nous étions à côté des Folies-Bergères, dans un appartement

JMA.- Pas mal..

MB.- Hé, hé.., alors dans cet appartement, bon, vous voyiez défiler des gens des sciences humaines, pour travailler avec nous. Du moment où nous avons été dans ce bâtiment-ci, çà a été fini.

JMA.- En quelle année?

MB.- 1970, 71, ce bâtiment a été construit à la fin des annés 1960.

JMA.- J'ai entendu que c'était pour la police

MB.- Non, pas pour la police, non.

JMA.- J'ai vu quelque bâtiment de la Police à Paris, avec la même architecture.

MB.- Peut-être ont-ils été faits par le même architecte. Autrefois, ce bâtiment était une prison militaire. Dreyfus a été emprisonné ici. Ensuite, c'est devenu une prison civile après la guerre. Le Ministère de la Justice a voulu garder des bureaux et une partie de ce bâtiment, et cette cohabitation avec le Ministère de la Justice a duré jusqu'en 1975-76.

JMA.- Alors, la situation était pire, quand tout le monde était ensemble?

MB.- Oui, c'est tellement plus simple de dire: «j'ai des données, et je ne réfléchis pas trop.» Avant, on le faisait faire par des esclaves, on avait des cartes perforées, le programme ne sort que des magnifiques nuages de points, on est content, et on ne réfléchit plus. 
JMA.- Faire çà à l'intérieur de l'université, c'est vraiment un problème, parce qu'il y a beaucoup de travail d'esclave. En Espagne, je ne sais trop pourquoi, il y a une relation importante avec les entreprises privées; çà, les gens le font pour des raisons purement économiques. J'ai entendu dire qu'il y a des monastères qui, à l'intérieur, font ce type de travail.

MB.- J'ai l'impression que maintenant, il y a un petit mouvement inverse qui commence à se dessiner. J'avais espéré qu'avec l'introduction des micro-ordinateurs, les gens introduiraient eux-mêmes leurs données, et que çà les pousseraient à réfléchir, mais pas du tout. Ils sont fascinés par l'aspect informatique des choses; alors, ma conviction profonde, c'est que vous ne pouvez avoir d'idées que si vous connaissez vraiment les données.

JMA.- Il faut faire toute l'enquête pour savoir ce qu'est une fiche. J'ai l'habitude de montrer l'ancienne fiche aux étudiants pour savoir ce qu'est un questionnaire et voir comment on fait la tabulation. En Espagne, la tabulation du questionnaire se fait sur le cadre de l'ancienne fiche perforée. S'ils connaissent l'histoire de la fiche, il leur est facile de comprendre la structure du questionnaire.

MB.- Les méthodes sous-jacentes des ordinateurs sont impénétrables pour la plupart des gens; en plus, il y a des erreurs et les gens ne s'en aperçoivent pas. J'ai vu des trucs monumentaux: des collègues en sociologie de l'éducation qui traînaient des erreurs de calcul, et ils ne se sont pas aperçu que c'était complètement faux. Les gens ne réfléchissent plus.

JMA.- Pour moi, çà continue à être un mystère, peut-être parce qu' aujourd'hui, par la publicité, les entreprises qui vendent des ordinateurs sont plus fortes, elles ont beaucoup de pouvoir.

MB.- Oui, oui, la méthode de Benzecri est très séduisante, avec sa philosophie; c'est un type très religieux, et sa philosophie c'est «l'harmonie dans les choses» -c'est Dieu qui l'a mise. Ce n'est pas la peine de faire des hypothèses, «l'harmonie est dans les choses». C'est l'époque de la bonne méthode scientifique..

JMA.- Et la méthode de Bourdieu?

MB.- Les gens de Bourdieu passent leur temps à utiliser les méthodes de Benzecri. Je leur dit toujours que les découvertes importantes en sciences sociales quantitatives ont été faites à l'époque de Quetelet ou Pareto, lorsqu'il n'y avait pas d'ordinateurs. 
JMA.- La polémique entre les sociologues qu'on appelle qualitatifs et ceux qu'on appelle quantitatifs; il y a eu une dispute très forte pendant les années 1960 entre l'Ecole de Francfort et les positivistes du Cercle de Vienne; elle s'est aujourd'hui atténuée, ou a presque disparue, et Armatte m'a dit quand je lui ai montré les papiers du congrès de Grenade: «J'ai trouvé que la dispute en Espagne est encore très forte entre sociologues qualitatifs et quantitatifs».

MB.- En France, il n'y a jamais vraiment eu dispute. Dès les années 1950-60, tout le monde avait compris que les mathématiques, dans leur rapport avec les sciences sociales ce n'est pas que du quantitatif. Lévy-Strauss disait: «le structuralisme n'est pas fait que de quantitatif, il a besoin de structure algébrique combinatoire.» Et même ici, on a toujours dit: «attention, les données statistiques en sciences sociales, ce n'est pas toujours du nombre», c'est souvent, par exemple, une échelle de préférence. On a essayé de développer des méthodes d'analyses ordinales de données. Je crois qu'il n'y a jamais eu de conflit parmi les gens qui disaient: «Attention une attitude scientifique et l'utilisation des méthodes mathématiques, çà a un sens dans les sciences sociales». Alors, le conflit, c'est plutôt — d'une part— avec les gens qui disent: «Non! Qu'on soit quantitatif ou pas, l'important c'est surtout: pas de mathématiques, pas de statistiques, pas de rationalité; pour traiter les problèmes des sciences sociales, il faut qu'on reste sur le plan subjectif et philosophique.» D'autre part, il existe une querelle politique entre l'école Boudon et l'école Bourdieu. C'est une question personnelle.

JMA.- En Espagne c'est un conflit avec les gens qui utilisent la statistique d'une façon mécanique, mais qui ont beaucoup de pouvoir à l'université et qui sont liés aussi aux entreprises privées. Le rapport entre les entreprises privées et la statistique en sciences sociales est très généralisé, le conflit ce n'est pas simplement entre l'école d'Ibañez et des gens qui font la statistique en étant liés à des entreprises privées. Je pense que la polémique en Espagne se situe entre comprendre les limites, ou travailler d'une façon critique; il faut penser les choses quand on utilise un programme statistique..

MB.- Oui, on a connu çà aussi en France..

JMA.- On peut dire que les courants qui utilisent la statistique d'une façon mécanique sont des courants influencés par les américains; les autres sociologues sont très influencés par la sociologie européenne, la philosophie, l'histoire, la linguistique.

MB.- Alors, là, à certains égards, on peut bien dire que la différence entre Bourdieu et Boudon est un peu de cette nature. Bourdieu, c'est l'utilisation purement mécanique, on passe des programmes et on n'essaie pas de savoir ce 
qu'il y a dedans, on s'en fout! Et puis Boudon, c'est l'ancienne manière, c'est essayer de fabriquer un modèle, essayer un modèle de causalité. Le reproche que je lui fais à Boudon, qui est un ami, c'est qu'il n'est pas mathématicien..

JMA.- Il n'est pas mathématicien?

MB.- Non, pas du tout-il en a juste fait un peu dans sa jeunesse-il fait des trucs qui le dépassent, et n'hésite pas à écrire et à publier.

JMA.- C'est le problème des gens qui écrivent beaucoup... Et l'histoire du Séminaire d'Histoire du Calcul de probabilités et de la Statistique, comment çà commence?

MB.- Dès la création de l'Ecole, il y a eu des rapports constants entre notre centre, ici, de Mathématiques sociales et le Centre Alexandre Koyré. C'est une vieille histoire. Pourquoi? Parce que-c'est un petit peu la philosophie pédagogique de Guilbaud-pour enseigner un morceau des mathématiques, il faut savoir d'où il vient, quels sont les problèmes qui se posent...

JMA.- A l'époque de Guilbaud, le Centre Alexandre Koyré était dirigé par René Tatom?

MB.- Alors, c'est çà, c'était l'époque où il était dirigé par Alexandre Koyré luimême, que j'ai connu, mais déjà il y avait René Tatom et Pierre Costabel, qui est mort maintenant et qui était un très grand ami de Guilbaud; ils se connaissaient depuis l'Ecole Normale. La collaboration se faisait principalement entre Costabel et Guilbaud. Costabel, sa spécialité était l'histoire des mathématiques aux XVII-XVIIIème siècles, et Guilbaud écrivait beaucoup sur Pascal. Ils avaient une collaboration très ancienne soit personnellement, soit dans les séminaires, dans les colloques. L'idée de ce séminaire, auquel vous assistez, remonte à 1982 avec Ernest Coumet, qui fréquentait le Centre de mathématiques sociales depuis très longtemps. C'est une connaissance très ancienne. On a monté ce séminaire parce que Coumet ne se sentait pas très à l'aise a l'époque, au Centre Koyré il y avait des problèmes. Pour les premières conférences, on a demandé à Bernard Bru et à Pierre Costabel, et ça continue, ça existe depuis 14 ans.

JMA.- Et les relations que vous avez eu avec Pareto?

MB.- Ah, Pareto! Il y a eu trois moments. Premièrement, j'ai été élève de Paul Levi en 1950. C'était un cours sur le mouvement brownien. Deuxième chose, à l'époque où je commençais au CNRS, Guilbaud faisait des séminaires sur la distribution Pareto. Il faisait des séminaires sur la distribution de Pareto; y est 
venu aussi Mandelbrot pour exposer ses travaux, aux alentours de 1960. Sous l'influence de Guilbaud, il y a eu des études empiriques dans les années 60 sur les revenus des professeurs pour voir s'ils s'ajustaient bien, la loi de Gibrat etc., des études comparatives; et puis des affaires de vocabulaire, les distributions de Pareto dans la distribution de vocabulaire, et çà a été fait sous la direction de Guilbaud, et de Madame Pétrochévith aussi.

JMA.- C'est la dame qui vient normalement au séminaire?

MB.- Oui, oui..

JMA.- Parce que j'ai rencontré Coumet et madame Pétrochévitch, un jour, devant le séminaire, et M. Coumet me l'a présentée.

MB.- Oui, c'est elle. Elle est à la retraite, mais elle continue de venir à des séances.

Çà, c'est la deuxième chose. A partir de 1983-84, j'ai commencé à m'intéresser de nouveau à Pareto comme vous avez dû le voir dans vos lectures, au moment où a ressurgi chez les sociologues français une grande querelle sur les inégalités, pour savoir si elles ont augmenté ou non dans l'enseignement depuis quelques années. C'est la polémique entre Boudon, Combessi, Gremi, les articles de la Revue Française de Sociologie. A ce moment-là, j'ai mis mon grain de sel en publiant un article utilisant les courbes de Lorenz. Çà m'a amené de nouveau à la distribution Pareto sur laquelle je n'avais pas travaillé depuis dix ans, et j'ai continué à faire des choses là-dessus parce que je trouvais çà intéressant.

JMA.- Bon, je ne sais pas s'il y a quelque chose que vous voulez ajouter...

MB.- Non, çà va.

JMA.- Á Madrid, peut-être. 